\title{
CARACTERIZACIÓN DE LESIONES HEPÁTICAS SÓLIDASCON TÉCNICA DE DIFUSIÓN POR RESONANCIA MAGNÉTICA: REPORTE PRELIMINAR
}

\section{Drs. María Loreto Vergara Del Río(1), Manuel Fernández $A^{(2)}$, TM. Rodrigo Pereira $B^{(3)}$.}

1. Médico Radiólogo Hospital La Serena, Alumna Magister UNAB-Clínica Las Condes, Santiago-Chile.

2. Médico Radiólogo, Clínica Las Condes, Santiago-Chile. Fundación López Pérez. Santiago Chile.

3. Tecnólogo Médico, Clínica Las Condes, Santiago-Chile.

CHARACTERIZATION OF SOLID LIVER LESIONS WITH DIFFUSIONWEIGHTED MRI TECHNIQUE

Abstract: The characterization of liver lesions in MRI is based on morphology, behavior in the various sequences, and with paramagnetic contrast. Objective: To evaluate the diffusion behavior of various solid lesions, measuring $A D C$ to determine its usefulness. Between 2007 and 2008 we studied 51 solid focal lesions (26 patients) with MRI, using conventional sequences and diffusion-weighted imaging obtained with EPI mode with different values of " $b$ ". Lesions corresponded to 20 hemangiomas, 12 focal nodular hyperplasia (FNH), 5 hepatocellular carcinoma (HCC), and 14 metastases. The statistical analysis allowed us to determine optimal cutoff level ( $A D C 1.28 \times 10^{-3} \mathrm{~mm}^{2} / \mathrm{s}$ ) to differentiate benign from malignant lesions. $A D C$ values of benign lesions were significantly higher than those of the malignant ones; the hemangioma was the solid lesion with higher $A D C$ values, followed by $F N H, H C C$ and metastases, with lower values. Registered mean values were 1.68, 1.30, 1.08 and 1.03 $\left(x 10^{-3} \mathrm{~mm}^{2} / \mathrm{s}\right)$, respectively.

In our view, the diffusion-weighted technique should be used to supplement conventional MRI for proper characterization of solid liver lesions.

Keywords: Diffusion, MRI, Solid liver lesions.

Resumen: La caracterización de las lesiones hepáticas en resonancia magnética se basa en: morfología, comportamiento en las diversas secuencias y con el contraste paramagnético. Objetivo: evaluar el com-

Vergara ML y cols. Caracterización de lesiones hepáticas sólidas con técnica de difusión por resonancia magnética. Reporte preliminar. Rev Chil Radiol 2010; 16(1): 5-10. Correspondencia: Dra. María Loreto Vergara del Río. loretovdr@gmail.com

Trabajo recibido el 15 de diciembre de 2009, aceptado para publicación el 26 de enero de 2010. portamiento en difusión de diversas lesiones sólidas, midiendo ADC para determinar su utilidad. En los años 2007-2008, se estudiaron 51 lesiones focales sólidas (26 pacientes) con resonancia magnética, utilizando secuencias convencionales y difusión, que fue adquirida con modalidad EPI con diferentes valores de " $b$ ". Las lesiones correspondieron a: 20 hemangiomas, 12 hiperplasias nodulares focales (HNF), 5 hepatocarcinomas (HCC) y 14 metástasis. El análisis estadístico permitió determinar nivel de corte óptimo (ADC 1.28 $\times 10^{-3} \mathrm{~mm}^{2} / \mathrm{s}$ ) para diferenciar lesiones benignas de malignas. Los valores $A D C$ de lesiones benignas fueron significativamente mayores que los de las malignas; el hemangioma fue la lesión sólida con mayor ADC, seguido por HNF, HCC y metástasis, con valores menores. Los valores medios encontrados fueron de 1.68, 1.30, 1.08 y $1.03\left(x 10^{-3} \mathrm{~mm}^{2} / \mathrm{s}\right)$ respectivamente. En nuestra opinión, la técnica de difusión debe utilizarse como complemento de resonancia magnética convencional, para una adecuada caracterización de lesiones sólidas hepáticas.

Palabras clave: Difusión, Lesiones hepáticas sólidas, Resonancia magnética.

\section{Introducción}

El hígado puede presentar una amplia variedad de lesiones sólidas, tanto benignas como malignas. La detección, caracterización y diferenciación certera de éstas ha sido siempre uno de los objetivos de los diferentes métodos de imágenes.

En resonancia magnética $(\mathrm{RM})$, la caracterización de estas lesiones se basa en su morfología, intensidad de señal en las diferentes secuencias (HASTE, T1) y en su comportamiento con contraste paramagnético (Gadolinio). También se han usado contrastes específicos que, dado su alto costo, no están disponibles en nuestro país. Sin embargo, aún con los protocolos habituales de estudio, que incluyen las secuencias antes mencionadas, todavía persisten casos donde no es posible lograr una adecuada diferenciación 
entre lesiones benignas y malignas

La técnica de difusión por RM, que no requiere uso de contraste, es ampliamente utilizada en el sistema nervioso central. Sin embargo, su utilización en abdomen sólo se describió en 1994 cuando Muller et al $^{(1)}$ presentan sus primeros trabajos en esta área. Desde entonces, se han publicado múltiples estudios sobre los parámetros técnicos ${ }^{(2-5)} \mathrm{y}$ las características de la difusión en los diferentes órganos sólidos tales como hígado, bazo, riñón y páncreas ${ }^{(6,7)}$, incluyendo enfermedades difusas y focales del hígado ${ }^{(6,8-10)}$. En nuestro medio no existen publicaciones en el uso de esta técnica en el estudio de lesiones hepáticas focales sólidas.

Las características de difusión en un tejido son evaluadas midiendo el Coeficiente Aparente de Difusión (ADC) que cuantifica el movimiento incoherente intravoxel, determinado por:

a) Perfusión capilar y

b) Movimiento browniano de las partículas de agua libre o difusión propiamente tal.

La difusión por RM refleja las propiedades de los tejidos, tales como amplitud de los espacios extracelulares, viscosidad, celularidad, integridad de membranas, etc.

\section{Objetivo}

El objetivo del trabajo fue evaluar las características de difusión de diferentes lesiones sólidas del hígado, cuantificando los valores de ADC para determinar si esta técnica es útil en la diferenciación de lesiones benignas y malignas.

\section{Materiales y Método}

Se revisaron retrospectivamente todos los estudios de RM de hígado realizados durante 8 meses (01 agosto 2007- 30 marzo 2008), midiendo los valores de ADC de las lesiones sólidas encontradas en el período.

El estudio de RM convencional y la difusión fueron realizados con un equipo Siemens modelo Avanto 1.5 Tesla, utilizando antenas spine matrix (columna) por posterior y body matrix (cuerpo) por anterior.

Todas las imágenes de difusión fueron adquiridas con modalidad Echo Planar Imaging (EPI) con técnica de adquisición en paralelo en plano axial, usando los siguientes parámetros: a) 12 a 18 imágenes para cubrir el hígado, b) tiempo de repetición (TR) de $1800 \mathrm{~ms}$, c) tiempo de eco (TE) de $85 \mathrm{~ms}$, d) matriz 192×192, grupo de visión (FOV) de $350 \mathrm{~mm}$, espesor de corte de $7 \mathrm{~mm}$ y $4 \mathrm{~mm}$ en lesiones pequeñas, f) distancia entre cortes de $0 \mathrm{~mm}, \mathrm{~g}$ ) factores de difusión ("b") de magnitud ascendente, es decir, con diferentes grados de sensibilización (50, 200, 400, 500, 700 y $850 \mathrm{seg} / \mathrm{mm}^{2}$ ).

La adquisición se realizó con 4 señales, y un tiempo total aproximado de 2:15 minutos, en respira- ción libre. Se utilizó técnica IPAT (Integrated Parallel Acquisition Techniques), en modo GRAPA (Generalized Autocalibrating Partially Paralell Acquisition) con factor de aceleración 2 y medición de antenas en modo dual.

Los estudios de RM convencional fueron interpretados por un médico radiólogo con dedicación en Imaginología Gastrointestinal con 10 años de experiencia en RM.

Las mediciones de ADC de todas las lesiones fueron realizadas en forma independiente por un médico radiólogo y un tecnólogo médico con dedicación en RM, promediándolas posteriormente. Para evitar errores de volumen parcial se seleccionaron sólo las lesiones sólidas iguales o mayores de $1 \mathrm{~cm}$ de diámetro, realizándose 3 mediciones por lesión con un área de muestra de $5 \mathrm{~mm}^{2}$, promediándolas para obtener el ADC final de cada lesión. En las lesiones con centro necrótico o fibroso se evitó medir esta área ( 5 casos constituidos por 1 hemangioma, 2 HNF y 2 metástasis). En aquellos pacientes que presentaban múltiples lesiones sólidas de similares características, se seleccionó al azar un máximo 3 lesiones.

El diagnóstico final de la naturaleza de las lesiones se determinó por sus características en: ultrasonografía (US), tomografía computada multicorte (TCMC) y RM sin/y con contraste ev, además de la evolución en los casos benignos (tiempo de control de 12 meses), los antecedentes de lesión primaria conocida y evolución en las metástasis. En los casos de HCC se consideró: la presencia de cirrosis más un nódulo con características propias de HCC en RM, nivel de alfafetoproteínas y/o biopsia.

Método estadístico: El análisis de los datos fue realizado con el software R (http://www.r-project.org/); toda decisión fue efectuada con un $95 \%$ de confianza. Los valores de ADC según la caracterización final de la lesión fueron comparados de acuerdo al procedimiento de Mann-Whitney. Se ajustó un modelo de regresión logística para modelar la probabilidad de que la lesión fuera maligna, ocupando el valor de ADC como variable predictiva. Con el modelo ajustado se determinó el punto óptimo de corte para la discriminación en términos de sensibilidad y especificidad.

\section{Resultados}

En el período 01 de agosto 2007 a 30 de marzo 2008 se estudiaron 97 pacientes con RM de hígado; en 46 de éstos se diagnosticó una o más lesiones nodulares sólidas hepáticas mayores de $1 \mathrm{~cm}$ Se descartaron 13 pacientes por no tener secuencias de difusión, 1 paciente cuyo diagnóstico final fue proceso inflamatorio focal que regresó con tratamiento antibiótico, 2 pacientes en que el nódulo correspondió a depósito focal de grasa, 1 paciente con diagnóstico de nódulo displásico sin confirmación y 3 pacientes con metástasis que habían recibido o estaban en 
tratamiento con quimioterapia o radiofrecuencia. De este modo, el grupo final estuvo compuesto por 26 pacientes con 51 lesiones nodulares sólidas: 11 hombres y 15 mujeres, con un promedio de edad de 51,5 años y un rango de 30-73 años.

Los diagnósticos finales de las 51 lesiones nodulares analizadas fueron: 20 hemangiomas, 12 HNF, 5 HCC y 14 metástasis.

El resumen de las mediciones de los valores de ADC obtenidos en cada tipo de lesión se muestra en la Tabla I y los box plots asociados se muestran en la Figura 1. En la Tabla I se observa que la distribución de los valores de ADC son ascendentes en el orden expuesto, evidenciando un significativo grado de superposición entre las mismas. En la Figura 1 se evidencia que en el grupo de HNF existen 2 obser-

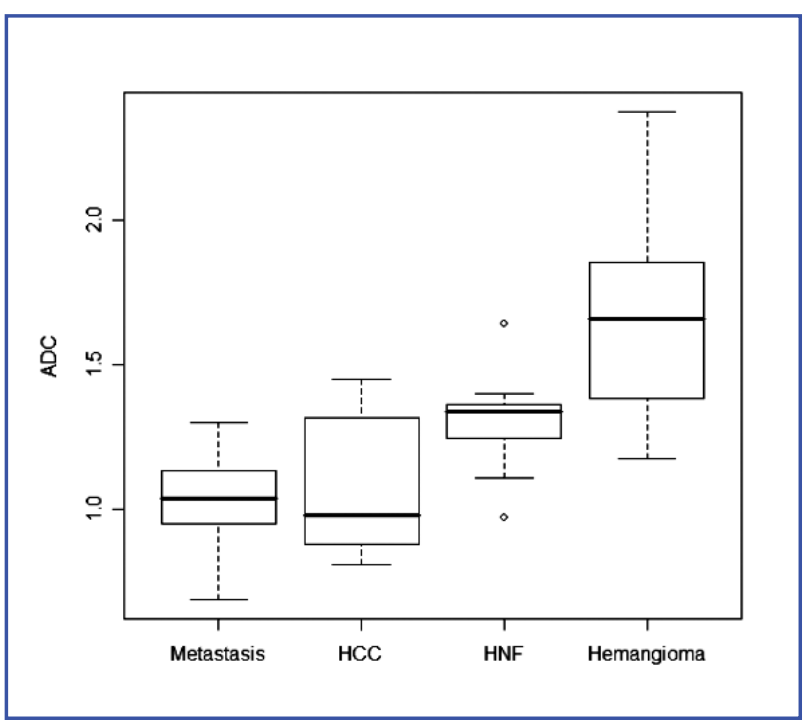

Figura 1. Box Plots de los valores de ADC en lesiones hepáticas sólidas. vaciones anómalas, que no siguen el comportamiento general del grupo; ésto puede ser explicado por el bajo número de observaciones y/o inexactitud de la medición del ADC.

La Tabla II muestra los resúmenes estadísticos de las mediciones de ADC de los diferentes tipos de lesiones, agrupadas en benignas o malignas. La media obtenida para las lesiones benignas fue de $1.54 \times 10^{-3} \mathrm{~mm}^{2} / \mathrm{s}$, lo que difiere significativamente de la media para lesiones malignas $\left(1.04 \times 10^{-3} \mathrm{~mm}^{2} / \mathrm{s}\right)$, según el test de Mann-Whitney $(p<0.05)$. La Figura 2 muestra box plots de los ADC para las mediciones de las 51 lesiones estudiadas, evidenciando que existe alto grado de superposición entre primer y último cuartil de las lesiones benignas y malignas respectivamente.

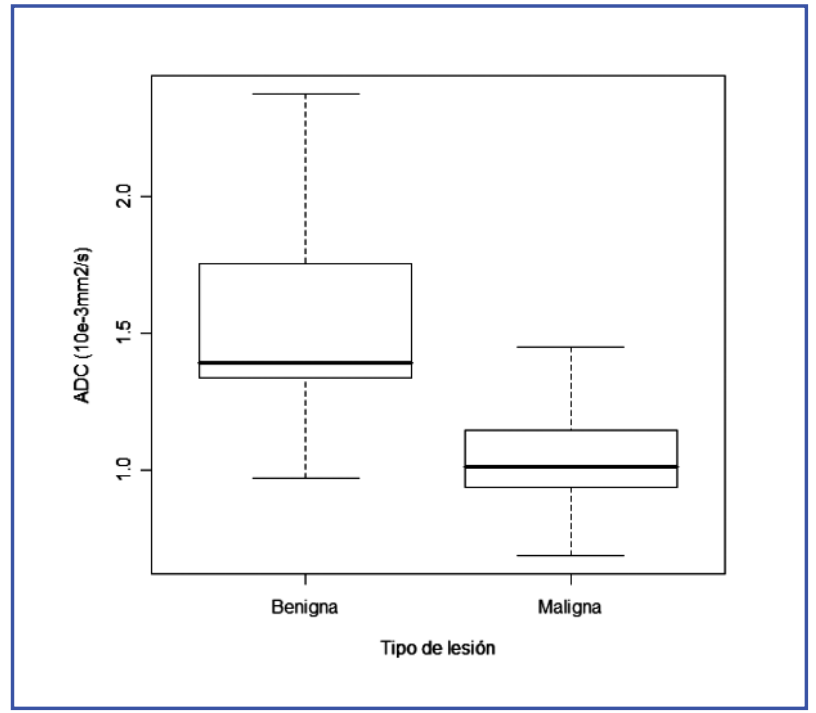

Figura 2. Box Plot de ADC en lesiones hepáticas benignas y malignas.

Tabla I. Valores de ADC en lesiones hepáticas sólidas.

\begin{tabular}{|lcccc|} 
Tipo de lesión & Media ADC & Desviación & Mínimo & Máximo \\
\hline Metástasis & 1.03 & 0.17 & 0.69 & 1.30 \\
HCC & 1.08 & 0.28 & 0.81 & 1.44 \\
HNF & 1.30 & 0.16 & 0.97 & 1.64 \\
Hemangiomas & 1.68 & 0.34 & 1.17 & 2.37 \\
\hline
\end{tabular}

Tabla II. Valores de ADC en lesiones hepáticas benignas versus malignas.

\begin{tabular}{|lcccc|} 
Tipo de lesión & ADC & D.S. & Mínimo & Máximo \\
\hline Benigna & 1.54 & 0.34 & 0.97 & 2.37 \\
Maligna & 1.04 & 0.19 & 0.69 & 1.44 \\
& & & & \\
\hline
\end{tabular}


Ajustando el modelo de regresión logística se buscó la mejor regla de decisión (nivel de corte) en base a la tasa correcta de clasificación. El valor de ADC obtenido fue $1.28 \times 10^{-3} \mathrm{~mm}^{2} / \mathrm{s}$.

La Tabla III muestra la relación entre las lesiones hepáticas benignas y malignas y la predicción de su naturaleza; fue realizada en base a un modelo con nivel de corte de ADC de $1.28 \times 10^{-3} \mathrm{~mm}^{2} / \mathrm{s}$. Con esta clasificación, se obtiene sensibilidad y especificidad de $84 \%$. La Figura 3 muestra el Box Plot de las lesiones benignas y malignas y su relación con el nivel de corte de ADC seleccionado $\left(1.28 \times 10^{-3} \mathrm{~mm}^{2} / \mathrm{s}\right)$

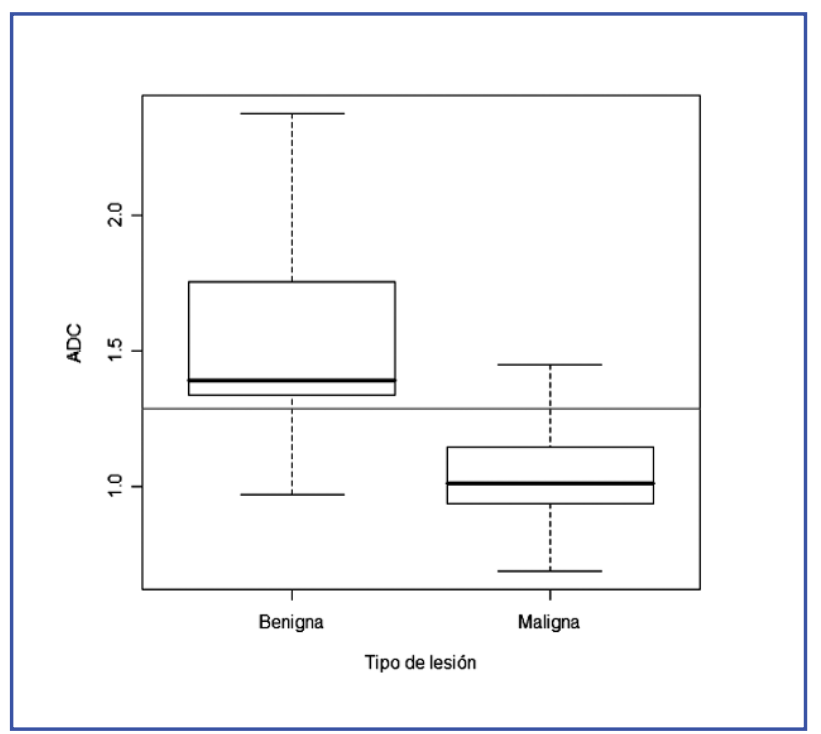

Figura 3. Box Plots de los valores de ADC en lesiones benignas y malignas en relación al nivel óptimo de corte.
En la Tabla IV se observa el comportamiento de los diferentes tipos de nódulos, estudiados en relación al nivel de corte utilizado $\left(1.28 \times 10^{-3} \mathrm{~mm}^{2} / \mathrm{s}\right)$. Si tomáramos como nivel de corte de $\mathrm{ADC}$ valores que nos permitieran un $100 \%$ de especificidad, veríamos que la sensibilidad con este valor sería muy baja. EI $100 \%$ de las lesiones con ADC $>1.44 \times 10^{-3} \mathrm{~mm}^{2} / \mathrm{s}^{(14)}$ correspondió a lesiones benignas (Figuras 4 y 5 ) (13 de 20 hemangiomas y 1 de $12 \mathrm{HNF}$ ) y el $100 \%$ con ADC $<0,97 \times 10^{-3} \mathrm{~mm}^{2} / \mathrm{s} \%{ }^{(7)}$ se trató de lesiones malignas (Figuras 6 y 7) (2 de 5 HCC y 5 de 14 metástasis). Entre estos dos grupos, encontramos superposición de valores en 28 lesiones, benignas y malignas ( 7 de 20 hemangiomas, 11 de 12 HNF, 3 de 5 HCC y 9 de 14 metástasis).

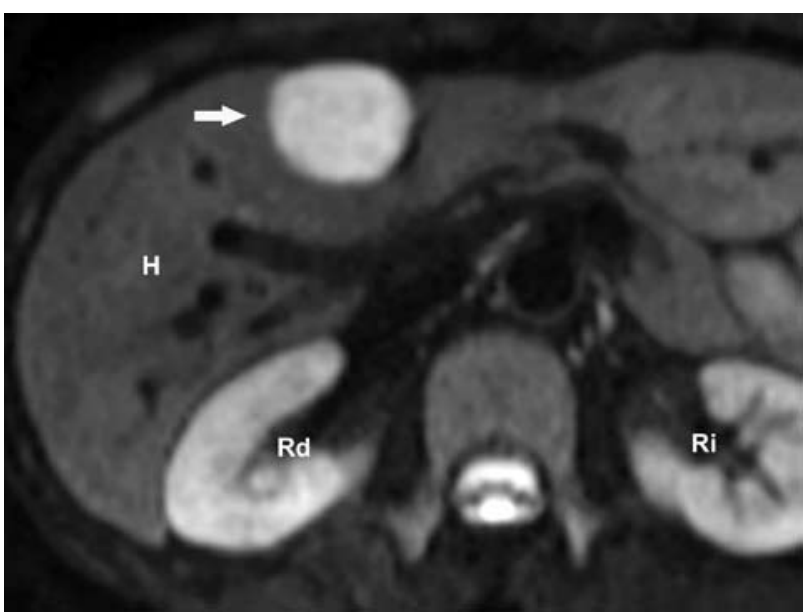

Figura 4. Estudio con difusión en un paciente con hemangioma. La imagen (flecha) es marcadamente hiperintensa indicativa de un ADC elevado, es decir, sin restricción importante al movimiento molecular. H: hígado, $R d$ y Ri, riñón derecho e izquierdo.

Tabla III. Relación entre naturaleza real y predicción de lesiones hepáticas.

\begin{tabular}{|llcr|} 
& & $\begin{array}{c}\text { Tipo de lesión } \\
\text { Benigna }\end{array}$ & Maligna \\
\hline \multirow{2}{*}{ Predicha por el modelo } & Benigna & 27 & 5 \\
& Maligna & 3 & 16 \\
& & & \\
\hline
\end{tabular}

Tabla IV. Distribución de lesiones hepáticas según nivel de corte.

\begin{tabular}{|lcccc|} 
Valor ADC & Hemangioma & HNF & HCC & Metástasis \\
\hline$>1.28 \times 10^{-3} \mathrm{~mm}^{2} / \mathrm{s}$ & 19 & 8 & 2 & 1 \\
$<1.28 \times 10^{-3} \mathrm{~mm}^{2} / \mathrm{s}$ & 1 & 4 & 3 & 13 \\
& & & & \\
\hline
\end{tabular}




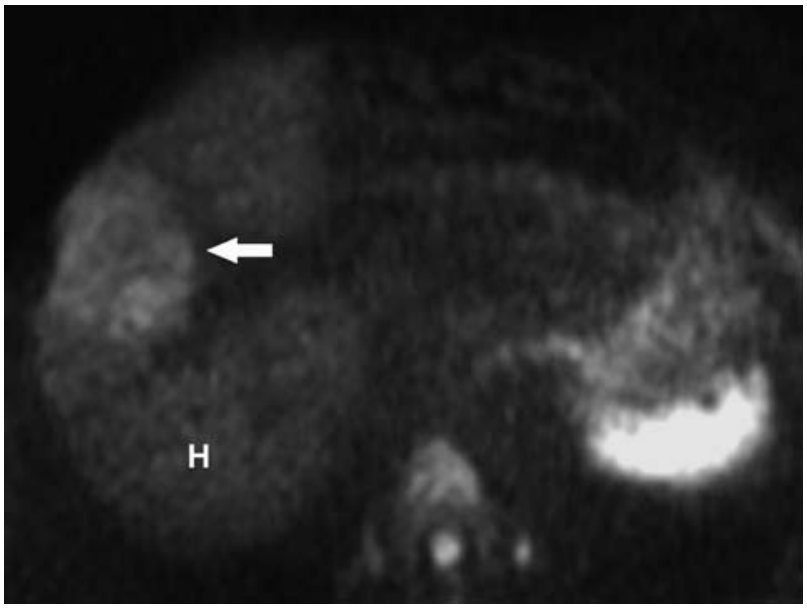

Figura 5. Estudio en paciente con HNF (flecha). La imagen es menos hiperintensa que en el caso de la figura 4, indicativa de una mayor restricción del movimiento molecular y por lo tanto un ADC menor. H: hígado.

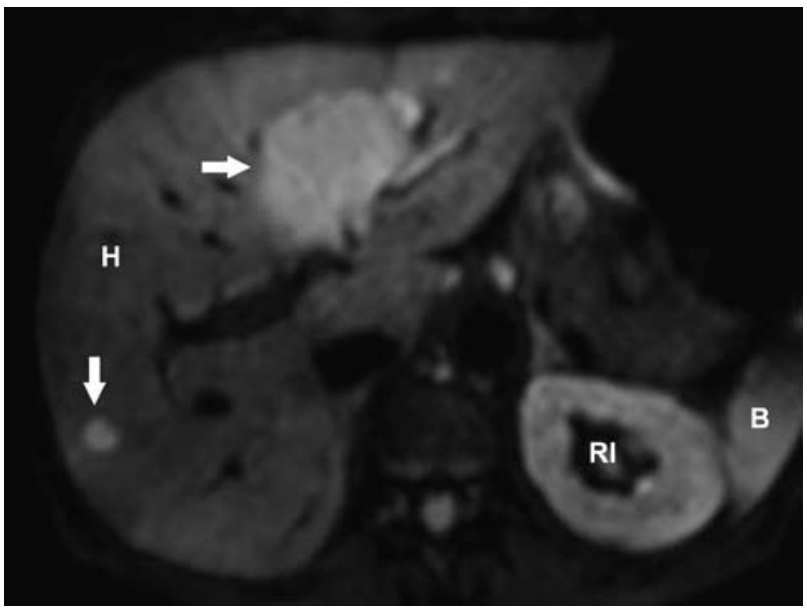

Figura 6. Estudio de difusión en paciente con metástasis (flechas). La restricción al movimiento molecular es levemente mayor (menor $A D C$ ) que en las lesiones benignas. $H$ : hígado, B: bazo, $R l$ : riñón izquierdo.

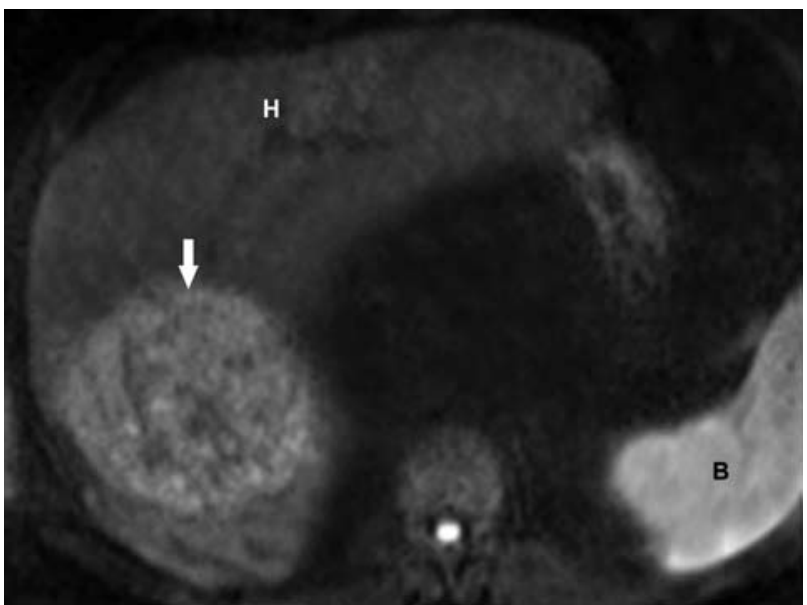

Figura 7. Paciente con HC. Al igual que en el paciente de la figura 5, la mayor celularidad de la lesión restringe el movimiento molecular, es decir, reduce el $A D C$. H: hígado, B: bazo.

\section{Discusión}

Publicaciones previas han demostrado la potencialidad del uso de ADC en la caracterización de lesiones hepáticas focales ${ }^{(3,7,8,9,10)}$ o difusas, en la detección de lesiones focales ${ }^{(11)}$ y en el control de tratamiento de tumores hepáticos primarios y secundarios $^{(12,13,14)}$.

Al igual que en la literatura existente, hemos encontrado que la técnica de difusión, utilizando como variable independiente el ADC, es útil en el estudio de las lesiones hepáticas sólidas para la diferenciación entre lesiones benignas y malignas, siendo el modelo estadísticamente significativo, con una sensibilidad y especificidad de $84 \%$ respectivamente. Este valor está limitado por la superposición de valores entre ambos tipos de lesiones, especialmente HNF y lesiones malignas (HCC y metástasis).

Si bien, en líneas generales, nuestros hallazgos sobre los valores de ADC de los diferentes tipos de lesiones son similares a lo encontrado en la literatura (los valores de los hemangiomas, son mayores que las HNF y estas mayores que los del HCC y de las metástasis) los valores absolutos no son iguales, lo que probablemente está determinado por diferencias en la técnica ("b" utilizados, respiración, forma de medición y técnica matemática utilizada).

Las limitaciones del estudio están dadas por el número de lesiones estudiadas y por no incluir la ubicación de las lesiones en los distintos segmentos del hígado, ya que hay publicaciones que han demostrado un diferente valor de ADC del parénquima hepático sano en algunos segmentos (ej. segmento VIII) susceptibles a la influencia del movimiento cardíaco y diafragmático y además por una menor relación señal/ruido en aquellos sectores más alejados de las antenas ${ }^{(15)}$.

El hecho de no haber incluido las zonas de necrosis ni fibrosis (2 metástasis, 2 HNF y 1 hemangioma) también debe ser tomado en cuenta en el análisis de los resultados, ya que estas zonas tienen un mayor movimiento de agua libre y por ende mayor ADC, lo que influye en las diferencias de valores de ADC reportadas por otros autores que han incluido estas zonas en su medición.

En conclusión, la difusión por RM es una técnica fácil de realizar, que no requiere contraste, agrega poco tiempo de exploración al examen y puede diferenciar lesiones hepáticas sólidas benignas de las malignas con una elevada sensibilidad y especificidad (84\%). Sin embargo, no ocurre lo mismo cuando se pretende diferenciar entre los diferentes tipos de lesiones sólidas benignas ni tampoco ayuda a diferenciar los diferentes tipos de lesiones malignas, ya que en estas situaciones existe un importante grado de superposición de valores de ADC.

En nuestra opinión, la técnica de difusión debe ser utilizada en el estudio de lesiones hepáticas sóli- 
das como complemento del estudio de RM realizada con secuencias convencionales, es decir, usada como una secuencia adicional al estudio habitual y no como secuencia única.

\section{Bibliografía}

1. Muller M, Prasad P, Siewert B, Niessenbaum M, Raptopoulos V, EdelmanR. Abdominal Diffusion Mapping with Use of a Whole-Body Echo-Planar System Radiology 1994; 190: 475-478.

2. Naganawa S, Kawai H, Fukatsu H, Sakurai Y, Aoki I, Miura $S$ et al. Diffusion-weighted Imaging of the Liver: Technical Challenges and Prospects for the Future. Magn Reson Med Sci 2005; 4(4): 175-186.

3. Yoshikawa T, Kawamitsu H, Mitchell D, Ohno Y, Ku Y, Seo $Y$ et al. ADC Measurement of Abdominal Organs and Lesions Using Parallel Imaging Technique. AJR 2006; 187: 1521-1530.

4. Nasu K, Kuroki Y, Sekiguchi R, Nawano S. The effect of Simultaneous Use of Respiratory Triggering in Diffusion-weighted Imaging of the Liver. Magn Reson Med Sci 2006; 5: 129-136.

5. Taouli B, Martin A, Qayyum A, Merriman R, Vigneron $D$, Yeh $B$ et al. Parallel Imaging and Diffusion Tensor Imaging for Diffusion-Weighted MRI of the Liver: Preliminary experience en Healthy volunteers. AJR 2004; 183: 677-680.

6. Kim T, Murakami T, Satoru T, Hori M, Tsuda K, Nakamura H. Diffusion-Weighted Single. Shot Echoplanar MR Imaging for Liver Disease. AJR 1999; 173: 393-398.

7. Yamada I, Aung W, Himeno Y, Nakagawa T, Shibuya $H$ Diffusion Coefficients in Abdominal Organs and Hepatic Lesions: Evaluation with Intravoxel Incoherent
Motion Echo-planar MR Imaging. Radiology 1999; 210: 617-623.

8. Bruegel M, Holzapfel K, Gaa J, Woertler K, Waldt S, Kiefer $\mathrm{B}$ et al. Characterization of focal liver lesions by ADC measurements using a respiratory triggered diffusion- weighted single-shot echo-planar MR imaging technique. Eur Radiol 2008; 18: 477-485.

9. Koh D, Scurr E, Pirgon A, Kanber B, Karanjia N, Brown $\mathrm{G}$ et al. Colorectal hepatic metastases: quantitative measurements using single-shot echo-planar diffusion- weighted MR imaging. Eur radiol 2006; 16: 1898-1905.

10. Sun X, Quan X, Huang F, Xu Y. Quantitative evaluation of diffusion-weighted magnetic resonance imaging of focal hepatic lesions. WJG 2005; 11: 6535-6537.

11. Nasu K, Kuroki $Y$, Nawano $S$ et al. Hepatic metastases: diffusion weighted sensitivity-encoding versus SPIOenhanced MR imaging. Rad 2006; 239: 122-130.

12. Deng J, Miller F, Rhee T, Sato K, Mulcahy M, Kulik L, et al. Diffusion-weighted MR imaging for determination of hepatocellular carcinoma response to yttrium-90 radioembolization. J Vasc Interv Radiol 2006; 17: 1195-1200.

13. Geschwind J, Artemov D, Abraham S. Chemoembolization of liver tumor in a rabbit model: assessment of tumor cell death with diffusion-weighted MR imaging and histologic analysis. J Vasc Interv Radiol 2000; 11: 1245-1255.

14. Deng J, Rhee TK, Sato KT et al. In vivo diffusion-weighted imaging of liver tumor necrosis in the VX2 rabbit model at 1.5 Tesla. Invest Radiol 2006; 41: 410-414.

15. Jones D, Basser P. "Squashing peanuts and smashing pumpkins": how noise distorts diffusion-weighted MR data. Magn Reson Med 2004; 52: 979-993. 